\title{
A new quantum model of the magnetic field of the Hot Earth, Moon and terrestrial planets
}

\author{
Vladimir Kuznetsov* \\ Institute of Cosmophysical Research and radio wave propagation FEB RAS, \\ 684034 Kamchatka, Paratunka, Mirnaya str., 7, Russia
}

\begin{abstract}
Distribution of P- and S- waves velocities in the Earth's inner core suggests that its matter has been quantum entangled since the origin of the Earth and the Solar system. This assumption we made allows us to develop the quantum model of the geomagnetic field evolution from its start to its disappearance. Unlike the generally accepted dynamo our model provides an obvious source of energy which is a phase transition and the thermal, mechanical and electrical energy released during it. The latter generates a double electric layer which rotation gives rise to the initial dipole field. Changing its direction the phase transition causes a reversal of the magnetic field. Magnetic and paleomagnetic data on the Earth, Moon, Mercury and Mars analyzed in the frameworks the Hot Earth model and features of their gravitation recorded at NASA project offered as conditions for the planets formation and evolution and so predictions for the further evolution of the Earth and its magnetic field.
\end{abstract}

\section{Introduction into the Hot Earth model}

The temperature $T$ at the centre of the star, planet, satellite at the time of their formation is the main parameter of our model. During evolution of the Solar system, the temperature estimated by: $T \approx 3 / 5 G M / R c_{p}$, where $c_{p}$ is the heat capacity of the substance, $G$ is gravity, and $R$ is the radius of the cosmic body is kept constant (somewhat decreased) only in the central part of the body. Temperature dependence on mass of a star, planet, satellite $T \sim M^{2 / 3}$ includes main-sequence stars, brown dwarfs, the planets of the Solar system and their seven large satellites. The temperatures estimated by this formula are somewhat overstated since the actual value of $c_{p}$ is increasing when temp's rising [1].

Here the planets and the satellites of the Solar system are qualified by their mass dependence of gravitational energy $W=3 / 5 G M / R(\mathrm{~kJ} / \mathrm{g})$, see, Fig. 1 . It should be noted that all bodies, ranging in size from the Sun to the Europa, a satellite of Jupiter, are spherical. The Europa's gravity potential $G M / R$ of about $2 \mathrm{~kJ} / \mathrm{g}$ along with the average value of its heat capacity $c_{p}=0.3 \mathrm{cal} / \mathrm{g}$. deg provides the temperature of its matter of about $1.5 \times 10^{3} \mathrm{~K}$. All other cosmic bodies including asteroids and satellites with their mass less than that of Europa are not spherical and apparently, they hadn't melted completely.

* Corresponding author: vvk@ikir.ru 
The heat of the evaporation (condensation) for silicon dioxide, as the main "building" material of terrestrial planets, is $U_{e}=15 \mathrm{~kJ} / \mathrm{g}$, and its heat of melting (crystallization) $\mathrm{U}_{\mathrm{m}}=1$ $\mathrm{kJ} / \mathrm{g}$. Divided into three classes the cosmic bodies at the Solar System are these with $G M / R$ $<U_{m}, U_{m}<G M / R<U_{e}$ and those objects that have $G M / R>U_{e}$. The small satellites and asteroids belong to the first class (I), the large satellites and the small (smaller than Venus) planets fall into the second one (II), and the planets with their mass greater than that of Venus are among the third class (III), see, (Fig. 1). Confirming $U_{1}$ and $U_{2}$ we access that Europe is the smallest spherical body and Titania is the largest non-spherical one. They differ ten times in mass and three times in size. The specific energy appears to be equal to the melting heat of the intermediate size cosmic body with $M \approx 2 \times 10^{25} \mathrm{~g}$ and $R \approx 10^{8} \mathrm{~cm}$, then $G M / R=1 \mathrm{~kJ} / \mathrm{g}$. According to our model the Earth's matter heated to high temperatures through the planet's formation had evaporated. Criterion for the total evaporation [2] is the compliance with the condition $G M / R=2 U_{\mathrm{e}}$ or $U_{e}=15 \mathrm{~kJ} / \mathrm{g}$.

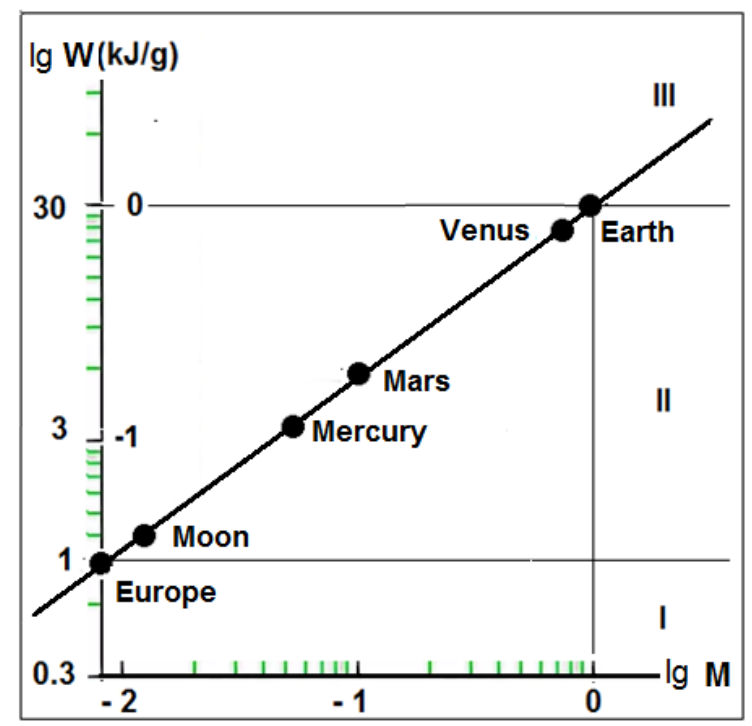

Fig.1. Specific gravitational energy $W$ of the planets related to the Earth is shown as function of their mass $M$. The bodies are divided into three classes: I, II and III.

Table 1. Planets parameters.

\begin{tabular}{|c|c|c|c|c|c|c|}
\hline & & $\mathrm{M} / \mathrm{M}_{\mathrm{Er}}$ & $\mathrm{R} / \mathrm{R}_{\mathrm{Er}}$ & $\mathrm{E} / \mathrm{E}_{\mathrm{Er}}$ & $\mathrm{p} / \mathrm{p}_{\mathrm{Er}}$ & $\mathrm{E} / \mathrm{U}_{\mathrm{m}} \quad \mathrm{E} / \mathrm{U}_{\mathrm{s}}$ \\
\hline 1 & Mercury & $5,5 \times 10^{-2}$ & 0,375 & 0,14 & 0,13 & 4.2 \\
\hline 2 & Mars & $10^{-1}$ & 0,53 & 0,19 & 0,14 & 6.0 \\
\hline 3 & Venus & 0,815 & 0,93 & 0,85 & 0,76 & 1.7 \\
\hline 4 & Earth & $1\left(6 \times 10^{27} \mathrm{~g}\right)$ & $1\left(6,4 \times 10^{8} \mathrm{~cm}\right)$ & $1(30 \mathrm{~kJ} / \mathrm{g})$ & $1(360 \mathrm{GPa})$ & 2.0 \\
\hline 5 & Moon & $1,2 \times 10^{-2}$ & 0,26 & 0,038 & 0,026 & 1.3 \\
\hline
\end{tabular}

The Earth's gravitational energy $E=3 / 5 G M^{2} / R=2.25 \times 10^{39} \mathrm{erg}$ (here: $G$ is gravity, $M$ - the Earth's mass and $R$ - its radius) [1] necessary to smash the Earth's mass into dusty particles far from each other would come out in the reversal process of the Earth matter compression by selfgravitation yielding its heating. Dividing $E$ by the heat capacity of the Earth's substance $\left(c_{p}=0.3 \mathrm{cal} / \mathrm{g} \cdot \mathrm{grad}\right)$ and its mass we see the temperature of the Earth's matter of about $30000 \mathrm{~K}$ [3]. Estimating the initial radius of the Earth $R_{\mathrm{o}}$ one can see that the 
continents can be laid one to another with virtually no gaps on the ball with the radius equal to that of the outer core of 3.5 thousand kilometers which was the Earth's initial radius.

Our model of the Hot Earth proceeds from the assumption which I couldn't not validate in my earlier research that at its beginning the Earth's matter was a tight ionized vapour (35 $\mathrm{g} / \mathrm{cm}^{3}$ ) which after its cooling became quasi-solid [4]. The fact that according to the model, the matter located in the inner core was really solid, did not serve to prove this indisputable fact. The problem why P-and S-waves don't accelerate as they approach the core center, since both the density of the core and its pressure increase became solved after I got acquainted with a relatively young concept of physics - the quantum entanglement (entanglements).

\section{Quantum entanglement}

By "Science in Focus "(according to the materials of BBC Science Focus, No. 2, 2014) the quantum entanglement as a new promising scientific direction was included into "20 GREAT IDEAS". Needing shocks and the research excitement the science is fueled by Great Ideas. At any time some of them are wrong, others lead to new breakthroughs. Science moves on formulating these ideas together with the attempts to refute them. By-products are the advances in technology and the new branches of science... Among these 20, quantum entanglement is N6.

The fact that it is a completely new trend in physics is indicated at least by the fact that it does not have an established Russian name. In English, everything is unambiguous: Quantum Entanglement. But in Russian... Quantum nonlocality, quantum entanglement, entangled quantum states... It seems that the term of quantum entanglement is gradually winning.

It is the quantum entanglement (QE) that forms the basis of quantum teleportation, quantum computer and quantum cryptography. By analogy I entitled the quantum geophysics (www.vvkuz.ru).

A question arises, why is the QE as a new area of physics, relevant to such longestablished field of science as the geomagnetic field. The geomagnetic field is well known to arise in a liquid iron core under the magnetic dynamo effect. The dynamo mechanism definitely applies to all other space objects that have (or used to have) a magnetic field, for example, to the Moon or Mars. One wonders why was the magnetic field on the Moon and Mars and why had it disappeared? Was there a magnetic field on Venus?

About 40 years ago, I became interested in the problems of the Earth physics and, of course, the nature of geomagnetic field (EMF). The first thing I did was to uniquely determine the location of the source of EMF generation. Using various methods, I confirmed that it is generated inside the F-layer at the boundary of the Earth's inner core (the green label in Fig. 2) rather than that is prescribed in [5]. Secondly, I ascertained that ionization, the formation of electric charges, their separation in the F-layer and daily rotation provide a weak dipole magnetic field which may be strengthened to the required value by the Hall dynamo. In the 80-90 years I proceeded from this idea, but the scientific community did not accept it. Recently I made sure that my concept is correct after I discovered that a non-dynamo mechanism for generating a geomagnetic field is considered in the encyclopedia of geomagnetism [6].

Solving problems in physics of the Earth and, especially, of its magnetic field, I was interested in why do the velocities of $\mathrm{P}$-and $\mathrm{S}$-waves in the inner core behave so (red lines in Fig. 2) as if they are not affected by the fact that the external pressure and the matter density increase towards the center of the G-core, which should cause an increase in the values of these velocities. Only if a matter is quantum entangled it does not obey the laws of classical physics. I made sure after I had published a dozen of papers about various effects in geophysics that have the QE (books, papers and reports on website www.vvkuz.ru). 


\section{Quantum phase transitions}

Quantum phase transformation is a matter transition from one quantum thermodynamic phase to another when external conditions change, and it occurs in the absence of thermal fluctuations, that is, at $T c \rightarrow 0$. Thus, the system is rebuilt under the influence of any nonthermal parameters (for example, they are pressure or magnetic field).

Quantum fluctuations controlled by non-thermal parameters, such as pressure or particles concentration, are responsible for the phase transition in a quantum-mechanical system.

As estimated here the temperature of the Earth's matter at the time of its formation was: $T=$ $30000 \mathrm{~K}$ which is higher than the critical point (for example, the critical point of Au is about $6000 \mathrm{~K})$. At the critical point (CP) the quantum entangled substance [7] in a state of the quantum vapour is able to huge density fluctuations, which ultimately lead to an increase in the concentration of $\mathrm{Au}$ and other precious metals [8].

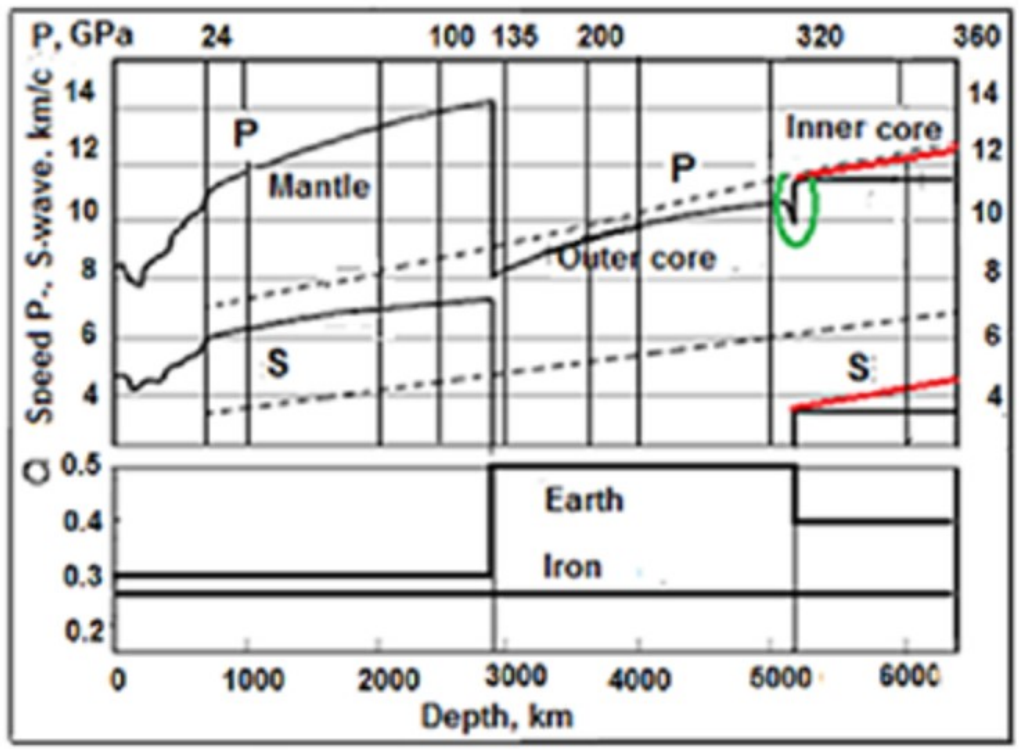

Fig. 2. The velocities of $\mathrm{P}$ - and S-waves in the Earth's shells and, in particular, in the G-core and in iron (dashed line) at the external pressure equal to that of the Earth's one. Poisson's coefficients $\sigma$ of Earth and iron [1].

\section{Generation of the geomagnetic field}

Fig. 3 shows the mechanism of the geomagnetic field generation with two modes in the F-layer which cause the Earth's expansion or its compression.

Condensation (C) of the superheated substance alternates its evaporation (E). Starting from the QE our concept of the geomagnetic field generation suggests that the acquisition of the $\mathrm{QE}$ (coherence) is replaced by its destruction (decoherence). The first mode results in the solid state of the Earth's substance (in the inner core or over the whole Earth just after its origin). The modes change if the internal core becomes overheated relative to the external one $\left(T_{G}>T_{E}\right)$ or backwards if $T_{G}<T_{E}$. 


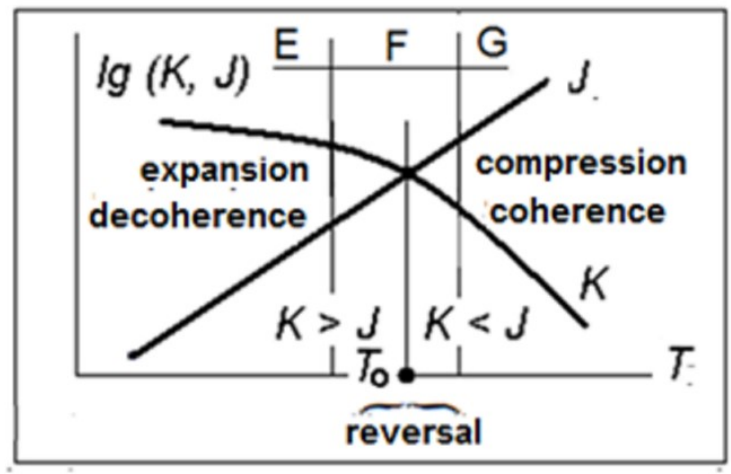

Fig. 3. Scheme of the phase transition in the F-layer after the classical model: $K$ - the rate of condensation of hot vapour, $J$-the rate of evaporation. Quantum entanglement considers the decoherence and coherence. Essentially there is matter compression or its expansion. When modes alternate, the reversal of the EMF occurs. Top: E-layer, F-layer, G-core.

Fig. 3 shows the change in the model parameters corresponding to the Earth's expansion (left) and the compression mode is given at the right. During the last 800 thousand years of Brunhes chron the Earth has been in the quiet period of its compression. Before Brunhes, the Earth was mostly expanding for one and a half million years of the Matuyama chron. Sooner or later, a period similar to the Matuyama chron (New Matuyama) will ensue [7]. This assumption will be discussed later, now we will justify the possibility for the EMF to arise without dynamo mechanism.

\section{Nondynamo theory}

At the encyclopedia of geomagnetism the nondynamo theories [6] offer four mechanisms: 1) Thermoelectric effect, 2) Thermomagnetic effect, 3) Hall effect and 4) Rotating electric field, based on: 1) Dipole associated with a rotating ferromagnet, 2) Differences in temperature and composition, 3) Currents arising in presence of the magnetic field, 4) Currents arising from the rotating charged capacitor.

The paper «Rotating electric fields and charges» ([5], P. 705, David J. Stevenson) placed here, states that EMF may be generated by the rotation of charges, the «problem is that in order to produce modest magnetic fields one requires very large voltage differences. The electric field $E\left(\mathrm{~V} \cdot \mathrm{m}^{-1}\right)$ required to provide a field $B(\mathrm{~T})$ is $\approx c^{2} B / v$, where $v$ is the rotational velocity. This suggests $E \approx 10^{11} \mathrm{~V} \cdot \mathrm{m}^{-1}$, which is physically impossible (it will ionize atoms) ». As one sees the generation of the EMF requires an electric field of a huge value. D. J. Stevenson is quite true if the emergence of a magnetic field in the Earth's core is carried out only by the rotation of electric charges. A weak dipole field generated by the daily rotation of charges needs to be amplified by a current of Hall type directed along that caused by the rotation of the double electric layer (DEL).

\section{Model parameters}

In the F-layer the electric field intensity $E_{R}: E_{R}=q /(C \times \Delta R)$, here $q=N e V$ - the charge of the layer, $\Delta R$ - its thickness, $N$ - the concentration of charges, $e$ - the electron charge, $1 / N e$ is the Hall constant $D, V$ - the volume of the spherical " capacitor": $V=4 \pi R^{2}{ }_{\mathrm{G}} \Delta R$, where $R_{G}$ is the radius of the inner core, then $C$ is the electric capacity of the DEL: $C=4 \pi \varepsilon_{0} R^{2} \mathrm{G} / \Delta R, \varepsilon_{o}-$ is the electric constant and $E_{R}=\Delta R /\left(\varepsilon_{o} D\right)$. 
As it is in the atmosphere the concentration of electric charges $\mathrm{Ne}$ seems to be unevenly distributed over the F-layer. As in the atmosphere clouds the charges separate in the region of the F-layer where condensation processes mainly occur resulting in the variation of $E_{R}$ over the layer (along $\Delta R$ ). For example, the electric field is maximum $\left(10^{5} \mathrm{~V} / \mathrm{m}\right.$ ) in clouds, where the generation of electric charges is commonly believed to occur. The field is high enough near the Earth's surface $\left(10^{2} \mathrm{~V} / \mathrm{m}\right)$ and it is much less in ionosphere. Our model suggests that $E_{R}$ should be maximal in thin layers at the boundaries of the F-layer, where, condensation and boiling are probably to proceed.

As discussed earlier, the daily rotation of the DEL with a frequency of $\omega$ may cause "quasicurrents " and generate the magnetic field. A positive charge is assumed here to be accumulated at one surface of the F-layer, and a negative - at the other one (Fig. 3). The Hall constant $D$ is positive in the first case $D^{(+)}$, and it is negative in the second one $D^{(-)}$.

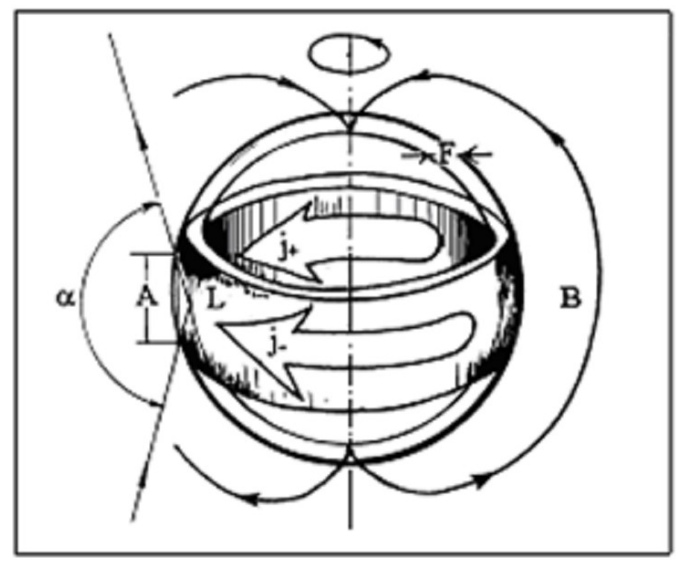

Fig. 4. Current rings at the F-layer [1].

The reversal of the DEL polarity, giving rise to the change of the "evaporationcondensation" mode, reverses the sign of $E_{R}$. The charges rotation at a velocity of $\omega R_{G}$ is equivalent to a "current" with a density of $j_{\omega}=\omega R_{G} / D$. The magnetic field (induction) of this "current" on the axis of the system rotation is:

$$
B=\mu_{o} j_{\omega} S_{X} / 2 R_{G}
$$

here $S_{X}$ is the effective area of charges assembling, $S_{X} \approx \delta \times A, \delta<<\Delta R$ is the thickness of the layer where the current flows $j_{\omega}$ ( $\mu_{0}$ - the magnetic constant, $A$ - the width of the layer). Since the charges at the boundaries of the F-layer are opposite in sign, there are two opposite directed currents (related to the rotation direction and the sign of $D$ ). At the center (on the axis of the Earth's rotation) of axial circular "currents" the magnetic induction is: $B=B_{G}-$ $B_{F}$, here $B_{G}=\mu_{o} j_{\omega} S_{X} / 2 R_{G}$, and $B_{F}=\mu_{o} j_{\omega} S_{X} / 2 R_{F}$. Substituting the corresponding values $j_{\omega}$ $=\omega R_{G} / D$ (in the second case, we use $R_{F}$ instead of $R_{G}$ ) we get $B=0$. However, this is not the case for points external to the current rings. For example, the value of the induction (the horizontal component of the field) at the equator is:

$$
B=\mu_{o} j_{\omega} S_{X} / h-\mu_{o} j_{\omega} S_{X} /(h+\Delta R) \approx \mu_{0} \omega S_{X} \Delta R R_{E} / h^{2} D,
$$

here $h=R_{E}-R_{F}, R_{E}$ - the Earth's radius. Substituting $\Delta R$ :

$$
B^{*}=\left(\mu_{o} \varepsilon_{o} \omega / R_{E}\right) \times S_{X} E_{R}
$$

The value of $B^{*}$ is determined by $E_{R}$ and the area occupied by the "current": $B^{*} \approx 10^{-28} E_{R} \times S_{X}$. Assuming $S_{X}$ of about $10^{9} \mathrm{~m}^{2}$ and taking into account that $E_{R}$ cannot be arbitrarily high, then the magnetic field generated by the rotation of the DEL is far less $\left(\mathrm{k}_{\mathrm{o}}>>1\right)$ than that required (at the equator the geomagnetic field $B \approx 3 \times 10^{-5} \mathrm{~T}, k_{o}$ is the required coefficient of the field 
increase). This assessment confirms the view of other authors that the geomagnetic field is impossible to be generated just due to the charges separation inside the DEL and its daily rotation.

The currents inside the F-layer are discussed here as those inside the spherical capacitor with two thin conductive layers-linings $\delta$ thick, here one of them is close to the inner core and the other one adjoins the outer core. Note that the current $j_{\omega} S_{X}$, induces a magnetic field $B_{o}$ in the area of the opposite layer - $\delta$ where the radial field $E_{R}$ acts and the current $I_{R}$ is directed normally to the field $B_{o}$. Generated by the co-influence of $B_{o}$ and the current $j_{R}$ the Hall one is directed alike that of rotating charges $j_{\omega}$ which may be amplified due to the Hall effect

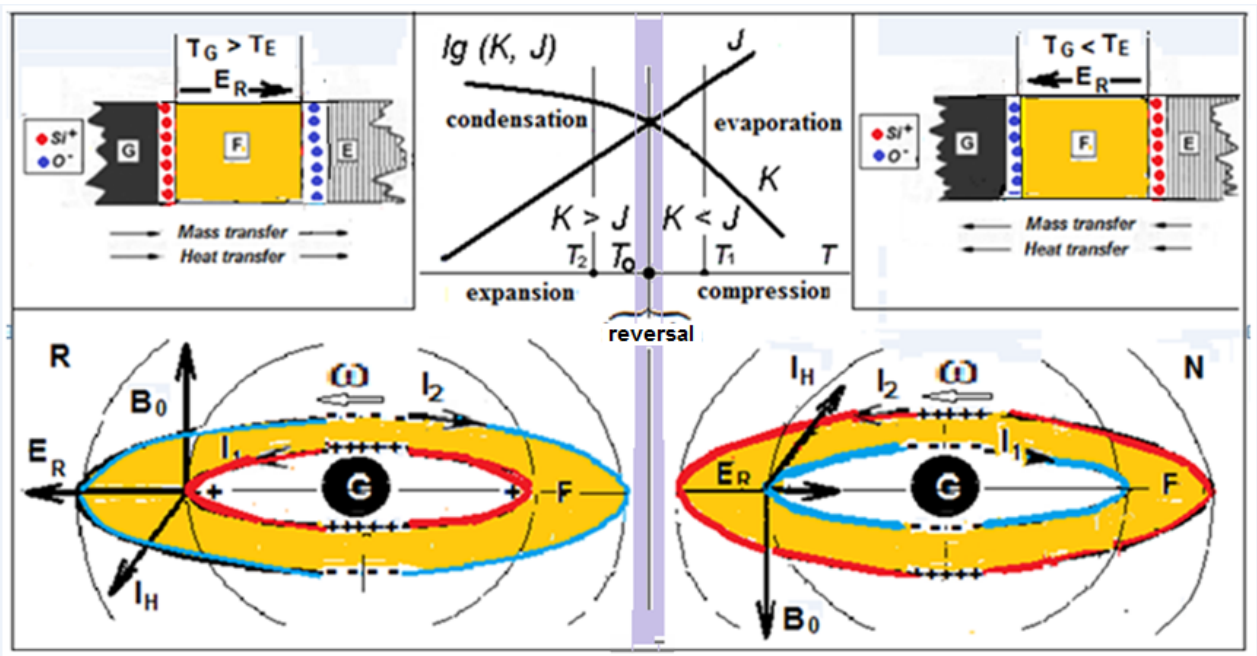

Fig. 5. Heat and mass transfer and charges separation in the F-layer. Left - the expansion mode (R), right - the compression mode $(\mathrm{N})$. The formation of DEL and its daily rotation giving rise to the initial field $B_{0}$. The interaction of $B_{0}$ with the radial electric field of the DEL $E_{R}$ generates the Hall current $I_{H}$ directed along the current producing the field $B_{0}$.

Let us estimate $B_{o}$ generated in the vicinity of a lining $\delta$ thick by the current $j_{\omega} S_{X}$ flowing along the other lining:

$$
B_{o}=(1 / 2 \pi) \mu_{o} j_{\omega} S_{X} / \Delta R=(1 / 2 \pi) \mu_{o} \omega S_{X} R_{G} / D \Delta R .
$$

Nearby the currents $j_{\omega}$ the directions and magnitudes of $B_{o}$ are the same because the direction of the current is determined by the direction of the Earth's rotation and the polarity of the electric charges or the Hall constant $D$.

The electric charges $D$, the radial electric field $E_{R}$, the current $I_{R}$ and the orthogonal magnetic field $B_{o}$ cause the Hall voltage $U_{X}=D B_{o} I_{R} / A$ and electric field $E_{X}=U_{X} / 2 \pi R_{G}$, as well as the Hall current $j_{X}$ :

$$
\begin{gathered}
E_{X}=D\left[B_{o} \times j_{R}\right], \\
j_{X}=\sigma_{X} E_{X},
\end{gathered}
$$

where $\sigma_{X}$ is the Hall conductivity, $j_{R}=I_{R} / S_{R}, S_{R}=2 \pi R_{G} \times A$. As it follows from our model, the Hall currents are opposite directed in $\delta$-layers where electric charges of opposite signs are concentrated. By our model a required field needs the current $j_{\omega}$ amplified by a factor of $k_{o}$. The possible value of $k$ follows from $k=j_{X} / j_{\omega}$. Substituting above mentioned formulas we get:

$$
j_{X}=\sigma_{X} D\left[B_{o} \times j_{R}\right]
$$


Let's derive $B_{o}$ from $j_{\omega}$ and $j_{R}$ from $E_{R}$ :

Then $k$ is:

$$
\begin{gathered}
B_{o}=\mu_{o} S_{X} j_{\omega} / 2 \pi \Delta R, \\
j_{R}=\sigma_{R} \Delta R / \varepsilon_{o} D .
\end{gathered}
$$

$$
k=\left(\mu_{o} / 2 \pi \varepsilon_{o}\right) \sigma_{X} \sigma_{R} S_{X}
$$

The current amplification due to the Hall effect is limited by the values of the conductivities $\sigma_{X}, \sigma_{R}$. To obtain the required magnetic field (induction), the obvious condition $k \geq k_{o}$ should be met. The coefficient $k_{o}$ should not be arbitrarily large, since it shows how much the velocity of the Hall current is greater than the speed of the charges daily rotation. The value of $k_{o}$ is bound by the current (ions moving) speed which is not high.

Estimating $\sigma_{X}$ and $\sigma_{R}$ in the frameworks of our model we assume that if $\sigma_{X}$ is large being close to the metal conductivity, then the small $\sigma_{R}$ is corresponding to the conductivity of dielectric. (The matter polarization and the formation of DEL are actually possible only in dielectric). Let's determine the electrical characteristics of our system with geomagnetic field generation. The amplitude of the Hall current $I_{X}$ and the voltage $U_{X}$ can be estimated using the restriction of the geomagnetic field power (dissipation) $P_{X}=10^{12} \mathrm{~W}$. Assuming that in the F-layer there are two currents opposite directed, and the induction $B$ in the region of the equator $(B \approx 30 \mu \mathrm{T})$ is:

$$
B=\mu_{o} I(1 / h-1 /(h+\Delta R)) \approx \mu_{o} I \Delta R / h^{2}
$$

then $I_{X} \approx 10^{10} \mathrm{~A}$ can be generated only if the inner core with its necessary ions charge is involved.

Similarly, the electrical characteristics of a system of radial currents and voltages can be evaluated. Apparently, the power of this system should be somewhat higher than that of the Hall one, since the Hall effect (if it is causing $I_{X}$ ) is secondary, but it should not be greater than the power of the heat flow $P_{R}=10^{13} \mathrm{~W}$ (we assume the converting efficiency of thermal energy into magnetic field energy of about $10 \%)$.

The electric current "space - the Earth" of (6.8-9.8) $10^{9} \mathrm{~A}$ is quite enough to generate the geomagnetic field. Reaching the Earth's surface, the current is most likely "washed away" into the space by the currents of calm rains.

\section{Evolution of the Earth's parameters in the context of our model of the EMF generation}

To date, geologists have collected samples, the processing of which shows that the earlier Earth used to have another composition and density of atmosphere, surface temperature, oceans, the speed of the Earth rotation, gravity and magnetic field, etc. Almost all of them are in contradiction with the model of the cold Earth. The known facts of the Earth's evolution are discussed here in the context of our model.

Fig. 6 [9] shows stages of the Earth's evolution: (1) - formed in a quantum entangled state as a ball with a radius equal to that of the outer core the Earth consisted of gravity-constrained shells of silicon oxide (basalt) with a density of $35 \mathrm{~g} / \mathrm{cm}^{3}$ and a temperature of $30000 \mathrm{~K}$ which is not observed now, (2) - loosing the QE through the first decoherence the matter (basalt) on the surface of the ball was heated (the red shell) to a high temperature, it evaporated then and during its further cooling turned into granite (Fig. 6) which formed the outer granite shell of the Earth. Escaped from the Earth the volatile impurities under the solar wind were split into hydrogen and oxygen, they combined then, rained and are preserved to our days in the form of fresh water, (3) - in the subsequent decoherences the liquid $\mathrm{E}$ was formed, where a layer of phase transition or the F-layer appeared, and the geomagnetic field was activated, (4) - after the repetitive decoherences, a liquid E-core and a solid D-mantle were formed, (5) - the inner G-core in the QE state is 
preserved inside the liquid core. After 5-10 million years, the G-core will melt due to the final decoherence and the geomagnetic field will disappear.

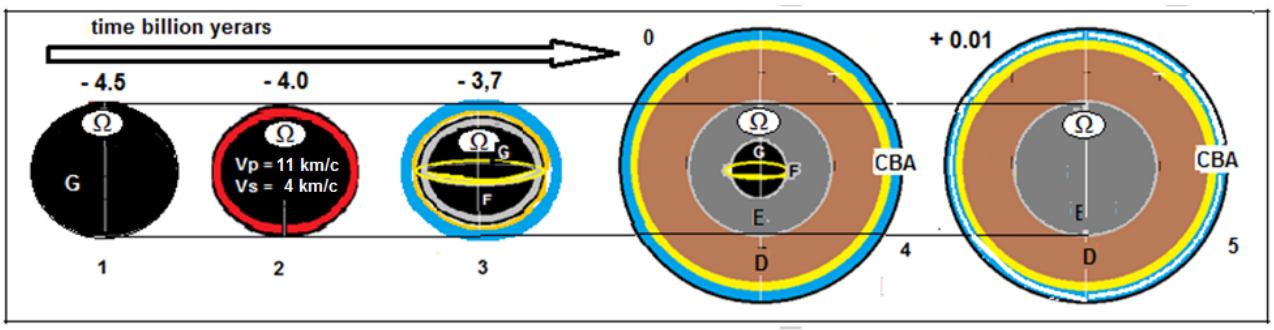

Fig. 6. Cooling of the hot, total quantum-entangled Earth from its formation 4.5 billion years ago up to nowadays approximated to the following 5-10 million years, after which the geomagnetic field will disappear.

\section{The first decoherence}

The velocities distribution of the seismic P - and S-waves inside the inner G-core and the Poisson's ratio (Fig. 2) related to the early period of the Earth's evolution, when all its matter was QE (Fig. 6-1, 6-2) provide the fact that in the inner core the wave velocities do not vary with the pressure increase along the radius of the Earth's core.
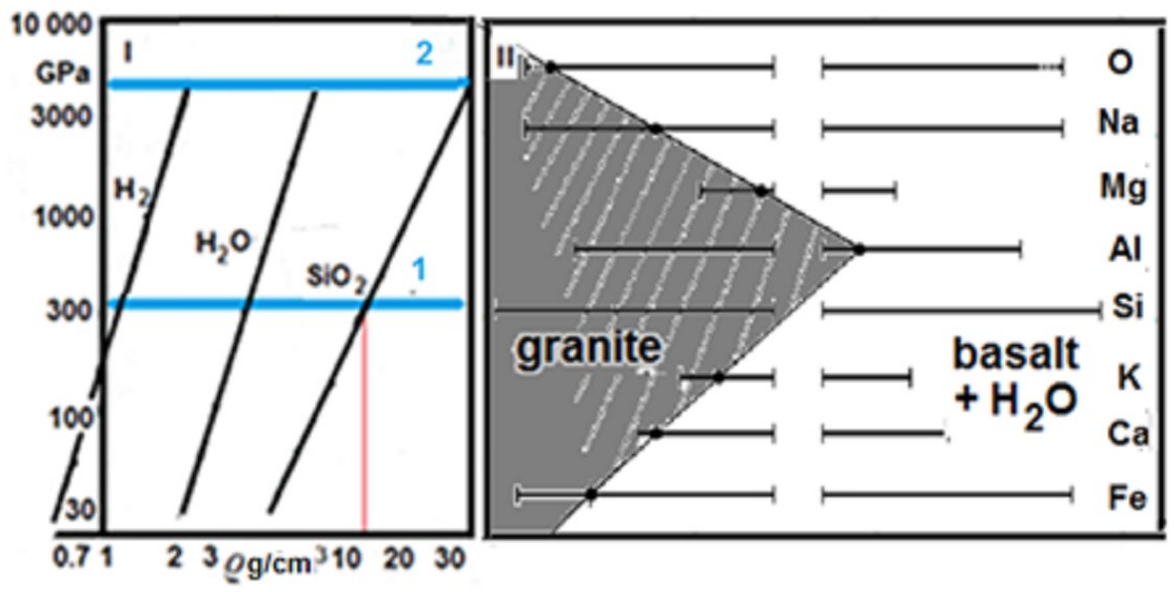

Fig. 7. I illustrates the main components of the Earth matter (pressure-depending density of $\mathrm{H}_{2}, \mathrm{H}_{2} \mathrm{O}$ and $\mathrm{SiO}_{2}$ ) [1]. Blue lines (BL): 1 - pressure in the center of the modern Earth, 2 - at the time of its formation. Fig. 7-II shows how one part of granite (grey) can be obtained from two parts of basalt [1]. The chemical elements of basalt are indicated at the right. The length of the strip shows the relative concentration of basalt. Relative concentrations of $\mathrm{Si}$ in granite and basalt are assumed to be equal. The concentration of $\mathrm{Al}$ in granite is greater than in basalt. If we assume that when heated, a part of basalt will evaporate, then the darkened part which is granite will remain.

During the Earth cooling, as the pressure inside the sphere surrounded by the granite shell had been decreasing the concentrations of hydrogen, water, and silicon oxide (basalt) had been changing (Figure 7-I).The fundamental difference between the Earth and other planets is the fact that the total matter of the Earth was quantum entangled. At the Moon and 
Mars, their quantum entangled matter was located in the center of the melt, which turned out at the Earth after a series of decoherences. It should be considered that the matter of the Earth had a high temperature and the traditional thermodynamics does not affect the quantum entangled matter. During the first decoherence the matter at the core boundary heated to a gaseous state was ejected into space, and the rest of it turned into the hot dense granite (Fig. 7-II), which formed a granite shell ten kilometers thick, where fresh water was raining. At the other planets where are no granites and fresh water this phenomenon did not occur.

\section{Confirmation of our model from the Kola Superdeep Borehole (KSB)}

The existence of granites and fresh water is known to be the main difference between the Earth and other planets of the Earth group. The Earth granites are wide described whereas I am not acquainted with any information about the origin of fresh water on Earth. There is no data why there are no granites (in large quantity) on the Moon, Mars. Here we discuss this fact in the frameworks of our model. The Russian physicist Ya. B. Zeldovich argued [3] his so-called "Principle of the total evaporation": the matter would evaporate entirely if the specific energy invested into it is twice the heat of the phase transition of evaporation. It is applicable to the Earth unlike other planets of the Earth's group. (The specific gravity energy of the Earth is $U=30 \mathrm{~kJ} / \mathrm{g}$, and the heat of evaporation of $\mathrm{SiO}_{2}=15 \mathrm{~kJ} / \mathrm{g}$ ). It follows that at the time of its formation the Earth's matter was entirely vaporized, otherwise, it was quantum entangled. The Moon's and Mars' matter was quantum entangled partly. Any breaking of the QE caused a huge heating of the Earth's matter: basalt, water, and other volatile components. The molten granite did not evaporate from the quantum entangled matter of the globe and cooling down it formed a granite shell as a base of modern continents.

The granite was produced from basaltic lava. The fact that in the absence of basalts the granites layer at the Kola Superdeep Borehole (KSB) was revealed caused a confusion among geologists. Nevertheless this fact proves our model.

A layer where the concentration of gold was elevated was found in granites at KSB. The Hot Earth model I am developing allowed me to tackle the problem how were mineral deposits generated. Basalt heated to about several tens of thousands degrees will turn into granite. If the temperature of granite is at the critical point, then, for example, gold at a temperature of about $6000 \mathrm{~K}$ acquires special quantum properties, called "giant density fluctuations", which lead to the fact that gold assembles into huge drops that will not disintegrate when cooling down [8]. This approach showed that temperatures and pressures at which diamond synthesis occurs were at a depth of hundreds kilometers at earlier stages of the Earth's evolution, whereas they correspond to ten kilometers at the depth of the modern Earth.

\section{The magnetic field of the Moon, Mars and Mercury}

Nature doesn't choose the mechanism of magnetic field formation for each planet aside. As Newton spoke such a waste is not inherent in nature. A person engaged into physics of a planet magnetic field does not worry that his model may not fit at another planet or satellite. Our model of the magnetic field of the Hot Earth can be legitimate only if it is applicable for every cosmic body. It is necessary to take into account that for a number of reasons the rates of planets and satellites evolution can vary greatly despite the fact that they were formed simultaneously in a single process. As follows from the Hot Earth model, our planet will soon spend the energy reserved in the inner core, and the geomagnetic field will extinct after further $2-3$ million years of its evolution. Venus, Mars, the Moon and other large satellites as opposed to Mercury are assumed to implement this script. 


\section{Features of the Moon's evolution}

Estimating the magnitude of the Moon's expansion we need the specific gravitational energy of the Moon: $e^{*}=1 / 2 G M / R=1.4 \mathrm{~kJ} / \mathrm{g}$. The heats of the phase transition (PT): $U_{2}$ is about 1 $\mathrm{kJ} / \mathrm{g}$ for melting-crystallization and $U_{I}=15 \mathrm{~kJ} / \mathrm{g}$ for evaporation-condensation. About 0.4 $\mathrm{kJ} / \mathrm{g}$ is left for the evaporation of the Moon's matter which is $0.4 / 15=3 \%$ of the Moon's mass. With Zeldovich criterion $\left(2 U_{l}\right)$ we get the half of the value that is $(1.5 \%)$ of the Moon's mass. Here we take $1.0 \%$. Since the Moon's mass is $7.3 \cdot 10^{25} \mathrm{~g}$ the mass evaporated is $\Delta M \approx 7 \cdot 10^{23} \mathrm{~g}$. The density of the Moon's matter is $3.5 \mathrm{~g} / \mathrm{cm}^{3}$ and its volume $\Delta V \approx 2 \cdot 10^{23}$ $\mathrm{cm}^{3}$. The Moon's surface $S=38 \cdot 10^{6} \mathrm{~km} \approx 4 \cdot 10^{17} \mathrm{~cm}^{2}$ and the increase of the Moon's radius $\Delta R=\Delta V / S=2 \cdot 10^{23} / 4 \cdot 10^{17} \approx 5 \mathrm{~km}$.

Our estimate was confirmed by the GRAIL mission [9] aimed at studying the gravity and the internal structure of the Moon resulted in comprehensive pattern of the lunar crust saturated with igneous intrusions which formed during the ancient lithospheric extension. The Moon was revealed [9] to have been expanding during the initial $700 \mathrm{Myr}$ of its evolution with the increase of lunar radius by $0.6-4.8 \mathrm{~km}$.

Given the volume of the Moon's evaporated mass and the diameter of its inner solid iron core equal to $480 \mathrm{~km}$, the initial density of the core matter was near to $10 \mathrm{~g} / \mathrm{cm}^{3}$ (Fig.8).

The initial density of the Earth was estimated earlier $(35 \mathrm{~g} / \mathrm{cm} 3)$ and the current one is $12 \mathrm{~g} / \mathrm{cm}^{3}$. The initial density of the hot cores of Mars and Mercury should be estimated from the relationship between the mass of the planet (satellite) and the initial density of its matter (Fig. 8).

Through the increase of lunar volume on $2 \cdot 10^{23}$ the density of its core had dropped by half resulting in $5 \mathrm{~g} / \mathrm{cm}^{3}$. It should be noted that as the Moon had been increasing due to conductive heat transfer its radius evolution follows $\Delta R \sim t^{1 / 2}$.

\section{The expansion of Mars and Mercury}

The average densities are: the Moon $-3.3 \mathrm{~g} / \mathrm{cm}^{3}$, Mars $-3.9 \mathrm{~g} / \mathrm{cm}^{3}$ and Mercury $-5.4 \mathrm{~g} / \mathrm{cm}^{3}$. Let's assume that after some expansion the Moon, Mars and Mercury lost their hot cores formed at the earliest stage of their evolution. It is assumed here that unlike Mercury the hot matter in the cores of the Moon and Mars has relaxed by our time. Then the average density of Mercury should be about $3.6 \mathrm{~g} / \mathrm{cm}^{3}$. The mass of the planets is taken not to change during their relaxation. Therefore, after the end of Mercury expansion its final volume should be near to $9 \cdot 10^{25} \mathrm{~cm}^{3}$, and its radius of 2.8 thousand $\mathrm{km}$ is more than the modern radius of Mercury by $360 \mathrm{~km}$, and the volume increased on $3 \cdot 10^{25} \mathrm{~cm}^{3}$ that is one and a half times of modern one.

$20 \%$ of Mercury mass $\left(6 \cdot 10^{25} \mathrm{~g}\right)$ has turned into gas. If the density of the gaseous substance was $15 \mathrm{~g} / \mathrm{cm}^{3}$ then the volume occupied by the core should be approximately $4 \cdot 10^{24}$ $\mathrm{cm}^{3}$, and the core radius should be $\approx 1000 \mathrm{~km}$. Assuming that $80 \%$ of Mercury mass remaining was concentrated in a space between the initial surface of Mercury and its core, the planet's density was about $3.6 \mathrm{~g} / \mathrm{cm}^{3}$ and the initial radius was near to $1800 \mathrm{~km}$. At its formation the density of the hot core of Mercury was about $15 \mathrm{~g} / \mathrm{cm}^{3}$ and the average density of the planet (Mercury) was slightly lower than the density of its core. During the evolution ( 4.5 billion years) the radius of Mercury has grown by about $600 \mathrm{~km}$ and its density has decreased to $5.4 \mathrm{~g} / \mathrm{cm}^{3}$. If the final density of Mercury's matter is $3.6 \mathrm{~g} / \mathrm{cm}^{3}$ then its radius will grow to 2.8 thousand $\mathrm{km}$. As one can see Mercury has to increase its radius on about 400 $\mathrm{km}$. It is difficult to estimate the time this process will take however it is clear that it should be close to that which has passed since the formation of the planet. It is obvious that the expansion of Mercury similar to that of the Moon follows: $\Delta R \sim \mathrm{t}^{1 / 2}$. 
During its formation about $50 \%$ of Mars' mass turned into gas which initial density is assumed to be $\sim 16 \mathrm{~g} / \mathrm{cm}^{3}$ (Fig. 8). Mars' mass is $6.4 \cdot 10^{26} \mathrm{~g}$, its radius is 3.4 thousand $\mathrm{km}$. We assume that the mass is equal to half of Mars' mass and the radius of its hot core is 1.7 thousand $\mathrm{km}$ that is close to that generally accepted $(1694 \mathrm{~km})$. (The radius of the core is believed to be about $1 / 3$ of the planet radius). Just like at Mercury another half of the mass occupies the space between the initial surface and the core.

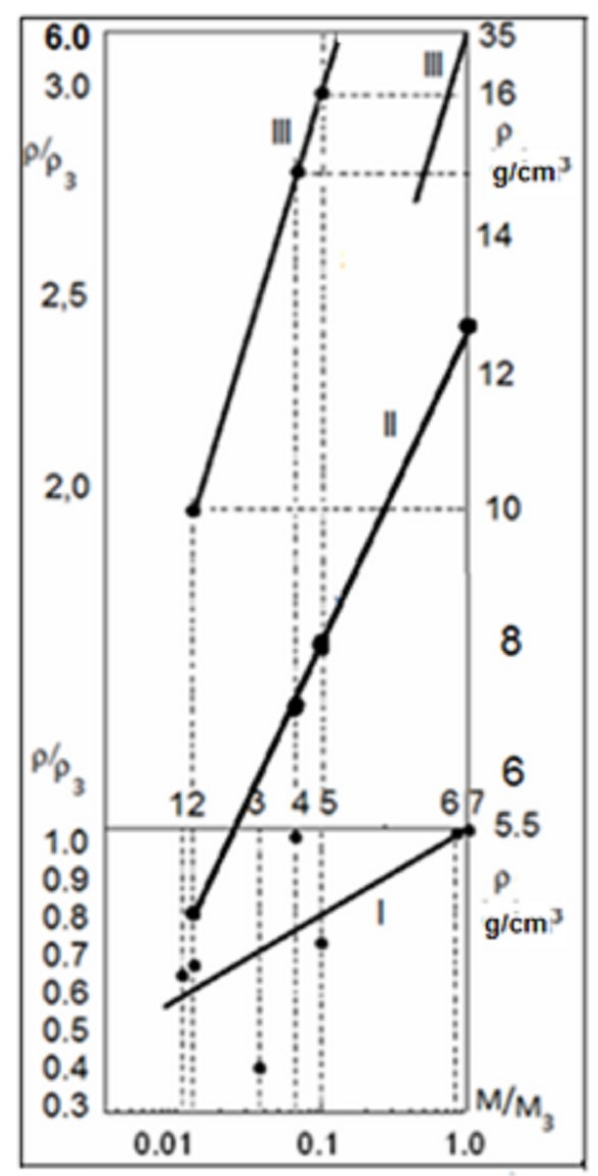

Fig. 8. Lines: I- the density of planets and satellites (right) and these relative to the Earth's mass (left). III - the density of gaseous matter at the time of planets and satellites formation. The density of the Earth and Venus is $30-35 \mathrm{~g} / \mathrm{cm}^{3}$, Mars- $16 \mathrm{~g} / \mathrm{cm}^{3}$, Mercury- $15 \mathrm{~g} / \mathrm{cm}^{3}$, the Moon- $10 \mathrm{~g} / \mathrm{cm}^{3}$. II - the density of cores matter after the expansion stage: 1 - Europa, 2- Moon, 3 - Ganymede, 4 -Mercury, 5- Mars, 6 -Venus, 7 - Earth.

If the matter density was $3.9 \mathrm{~g} / \mathrm{cm}^{3}$ then the initial radius of Mars was about 2.9 thousand $\mathrm{km}$. It follows that during its evolution Mars' radius has increased $\left(\Delta R \sim \mathrm{t}^{1 / 2}\right)$ on about $500 \mathrm{~km}(3.4-2.9)$, and the upper mantle composed of olivine $1113 \mathrm{~km}$ thick also corresponds to our result: $1200=2900-1700 \mathrm{~km}$. The thickness of the lower mantle is considered to be $561 \mathrm{~km}$, which is also close to our estimate $(3400-2900 \mathrm{~km})$. It should be noted that the expansion of Mars due to the decompression of its matter inside the inner core occurred at the boundary of its hot and dense core, i.e. in the lower mantle. The convection did not arise here as well. 
Nowadays Mars has finished its evolution with its radius of $3400 \mathrm{~km}$. Mercury is at the middle stage of its evolution and its current radius is $2400 \mathrm{~km}$. Mercury will expand further until its radius reaches $2800 \mathrm{~km}$. At the end of Mercury's evolution the density of its matter intermediate between those of the Moon and Mars will be $\approx 3.6 \mathrm{~g} / \mathrm{cm}^{3}$.

Silicon oxide as the operating substance of the planets was useful because the behavior of the $\mathrm{SiO}_{2}$ density as a function of pressure is well known, and we used this simplification when we were discussing the Earth. The condensation of $\mathrm{SiO}_{2}$, its relaxation and transition into a condensed (liquid) state and, ultimately, the decompression of the compressed substance and planets expansion is a source of heat flow, tectonic phenomena and the energy necessary to generate the planet's magnetic field. In our model all these features are interrelated. The pressure at the center of Mercury and Mars is close to 0.13 and 0.14 of that at the center of the Earth (3.6 Mbar) or 0.4 and 0.5 Mbar. The corresponding density at the center of the planets, if they consist of $\mathrm{SiO}_{2}$ (Fig. 7), is approximately $7-8 \mathrm{~g} / \mathrm{cm}^{3}$.

The density of the Earth's substance at the inner core is known to be about $12 \mathrm{~g} / \mathrm{cm}^{3}$. The initial density of the recompressed gaseous substance of the Earth was $35 \mathrm{~g} / \mathrm{cm}^{3}$. In the process of evolution the gaseous substance had been cooling and condensing, its pressure $p$ and density had been decreasing (at the initial stage $p \sim T$ ). For example, at the time of the Earth formation its pressure was about 15 times higher than the modern one.

As shown above the initial density of the lunar inner gaseous core was about $10 \mathrm{~g} / \mathrm{cm}^{3}$. Let's assume that the difference between the planets (at least, of the Earth's group) including the density of their gaseous substance is determined by the magnitude of their mass. If we draw a straight line on the density - mass scale from 10 to $35 \mathrm{~g} / \mathrm{cm}^{3}$ (Fig. 8) it turns out that the density of the gaseous substance in the inner core of Mercury is about $15 \mathrm{~g} / \mathrm{cm}^{3}$, and that of Mars is $16 \mathrm{~g} / \mathrm{cm}^{3}$.

Should be repeated that the Mercury magnetic field indicates that its inner core had the initial density of about $15 \mathrm{~g} / \mathrm{cm}^{3}$ and it is noticeably lower now. The Mars' core functioning at a certain stage of its evolution which lasted according to various estimates from 1 to 2 billion years had already eliminated its energy reserve.

\section{Tidal effects and slowing of Mercury's rotation}

Powerful solar tides had reduced the speed of Mercury rotation from 8 hours up to 58.65 earth days. Due to the tidal braking as it was moving away from the Sun, Mercury was changing its angular velocity of rotation. Complete rotation around its axis Mercury makes in $2 / 3$ of its year. The duration of Mercury sunny day is 176 earth days. It takes 87.969 earth days to orbit round the Sun. Mercury makes three rotations around its axis in two Mercury's years. Similar to the Moon the speed of Mercury's rotation around its axis should coincide with that of its rotation around the Sun.

The rotation of the Earth is too decelerated by the tidal friction, and the duration of the day gradually increases. Over the last 2.5 thousand years, it has increased by about 0.0024 seconds per century. Let's estimate the tidal effect for Mercury. If the initial period of Mercury's rotation around its axis was about 8 hours and after 4.5 billion years the modern one is 58 days then the average deceleration rate of Mercury is approximately 0.1 seconds per 100 years, that is about 100 times higher than that of the Earth.

Due to the tidal friction the rotational speed of Mercury decreased by 175 times. The rotational energy of Mercury at the initial stage of its evolution was $E \omega=M(\omega R)^{2} / 2=7 \cdot 10^{26}$ $\mathrm{J}$ and the specific rotational energy was $\approx 2 \mathrm{~kJ} / \mathrm{g}$. The specific rotational energy of modern Mercury is $(175)^{2} \approx 3 \cdot 10^{4}$ times less implying that almost all the rotational energy has been spent during the evolution of Mercury. 

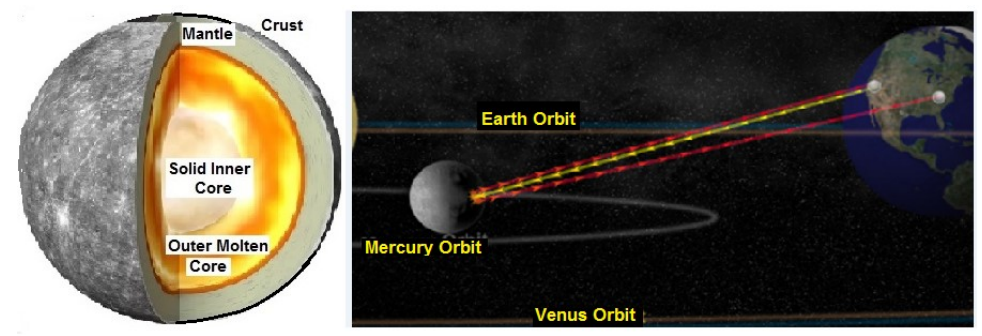

Fig. 9. The internal structure of Mercury (left) obtained by [10].

As estimated above, the evaporation and overheating of Mercury's matter should be spent to $\approx 3 \mathrm{~kJ} / \mathrm{g}$ (see Fig. 1 ) which is close to $E_{l}$. It is clear that during its evolution Mercury had dissipated its rotational energy close to that necessary to expand its radius, generate its magnetic field and heat flow. The tidal dissipation increased the temperature of the upper layers at Mercury's mantle and since the temperature gradient dropped the thermal outflow from the hot core decreased. The heat flow is provided by the condensation of the gaseous matter and its further crystallization into mantle. The Moon and Mars are cooling without additional heating, their gaseous matter has already relaxed, and the generation of their magnetic field has stopped. Mercury, Mars and the Moon unlike the Earth and Venus had been cooling due to the thermal conductivity which is characterized by the conductive heat transfer with $\Delta R \sim t^{1 / 2}$. (Here $t$ is the time of the planet's evolution). After the thickness of the liquid core at the Earth and Venus became more than $300 \mathrm{~km}$ the convection mode appeared, and further cooling was faster than $\Delta R \sim t^{1 / 2}$. This phenomenon did not occur at the Moon, Mercury and Mars.

\section{Conclusions}

A new model of the magnetic field of planets and large satellites is proposed. Unlike the generally accepted dynamo, our model proposes an obvious source of energy, it is a phase transition and the thermal, mechanical and electrical energy released during it. The last gives rise to a double electric layer, the rotation of which ensures an initial dipole field and the change of the phase transition mode causes a reversal in the polarity of the magnetic field.

In the frames of our model, such world problems of geomagnetism as reversals, the correlation between reversals and the intensity of tectonic phenomena, for example, the wellknown paradox "superchron - superplume, the source of the geomagnetic field generation found a simple unambiguous solution. The lack of clear answers to these questions result in misunderstanding of such phenomena as: why was there a field at the Moon, and why did it disappear? Why does the same situation about the magnetic fields of other planets and satellites exist? Why has Mercury a dipole field? Is there any data on the paleomagnetic field of Venus?

\section{References}

1. V.V. Kuznetsov, Vvedenie v fiziku goryachei Zemli (KamGU, PetropavlovskKamchatsky, 2008) (in Russian)

2. Ya.B. Zeldovich, Yu.P. Raizer, Physics of Shock Waves and High-Temperature Hydrodynamic Phenomena (Nauka, Moskow, 1966) (in Russian)

3. V.A. Magnitsky, Vnutrennee stroenie i fizika Zemli (Nedra, Moskow, 1965) (in Russian) 
4. V.V. Kuznetsov, E3S Web of Conf., 196 (2020)

5. V. V. Kuznetsov, Vestnik KRAUNC. Phys.-Mat. Science, 2, 9 (2014) (in Russian)

6. Encyclopedia of Geomagnetism and Paleomagnetism. Ed. D. Gibbins and E. HerreroBervera (Springer, Dordrecht, 2007)

7. V.V. Kuznetsov, Vestnik KRAUNC. Phys.-Mat. Science 35, 2 (2021) (in Russian)

8. V.V. Kuznetsov, Geologia i MSR Sibiri 3, 23 (2015) (in Russian)

9. J.C. Andrews-Hanna et al., Science, 339 (2013)

10. J. L. Margot et al., Science, 316 (2007) 